\title{
Intermediary Equatorial Orbits of an Artificial Satellite ${ }^{1}$
}

\author{
John P. Vinti \\ (October 27, 1961)
}

\begin{abstract}
A previous paper derived the solution for the drag-free motion of an artificial satellite in the gravitational field of an oblate planet. The corresponding potential, expressed in oblate spheroidal coordinates, leads to separability and represents the even zonal harmonics exactly through the second, for any oblate planet, and approximately through the fourth, in the case of the earth.

The previous paper contained a restriction on the orbital inclination $I$, viz, $I_{c}<I<180^{\circ}$ $-I_{c}$, where $I_{c}$ might be as large as $1^{\circ} 54^{\prime}$ for an orbit sufficiently close to the earth. The present paper removes this restriction and shows that many of the formulae for the periodic terms may be simplified, when the orbit is equatorial or almost so. The results agree with those obtained by a direct two-dimensional solution, when the orbit is purely equatorial.
\end{abstract}

\section{Introduction}

This paper is a sequel to a recent paper, ${ }^{2}$ concerning an accurate intermediary orbit for satellite astronomy, and will accordingly follow the notation thereof. It there followed that if

$$
\lambda \equiv b_{1} / b_{2}<1,
$$

all the $\rho$-integrals are expressible in terms of rapidly converging series involving products of Legendre polynomials with arguments $\lambda$ and $\left(1-e^{2}\right)^{-\frac{1}{2}}$. Condition (1) is equivalent to a restriction on the orbital inclination $I$, viz,

where, to the first order in $k$,

$$
I_{c}<I<180^{\circ}-I_{c},
$$

$$
\tan ^{2} I_{c}=k \equiv\left(r_{e} / p\right)^{2} J_{2} .
$$

For the earth $J_{2}=0.00108$, so that for orbits so close that $p \approx r_{e}$,

$$
I_{c}=1^{\circ} 54^{\prime} .
$$

I imposed the condition $\lambda<1$ in order that

$$
\left(1+A \rho^{-1}+B \rho^{-2}\right)^{-\frac{1}{2}} \equiv\left(1-2 \lambda h+h^{2}\right)^{-\frac{1}{2}}
$$

should be a generating function for the Legendre polynomials $P_{n}(\lambda)$. It now appears that such a restriction is unnecessary. Thus

$$
\left(1-2 \lambda h+h^{2}\right)^{-\frac{1}{2}}=\sum_{n=0}^{\infty} h^{n} P_{n}(\lambda)
$$

even when $\lambda \geqq 1$, provided only that ${ }^{3}$

$$
h<\lambda-\left(\lambda^{2}-1\right)^{\frac{1}{2}}
$$

We need show only that (7) is always satisfied, in order that all the results of (A) hold for all orbital inclinations. The only changes will be a few simplifications, especially for the cases $I=0^{\circ}$ or $180^{\circ}$ of purely equatorial orbits.

1 This work was supported by the U.S. Air Force, through the Office of Scientific Research of the Air Research and Development Command.

2 J. P. Vinti, J. Research NBS, 65B (Math. and Math. Phys.), 169-201 (1961), hereafter referred to as (A). Any reference in the present paper to an equation with a decimal number, such as (5.30), denotes an equation in (A).

3 E. W. Hobson, The Theory of Spherical and Ellipsoidal Harmonics, p. 15, Cambridge University Press, Cambridge 1931. 


\section{The $\rho$-Integrals}

To show that $(7)$ is always satisfied when $\lambda \geqq 1$, note that

$$
\frac{b_{1}}{\rho}=\frac{b_{1}}{b_{2}} \frac{b_{2}}{\rho}=\lambda h \text {. }
$$

By (4.12), (4.13), and (5.6) it follows that $b_{1}$ and $b_{2}$ are both real and non-negative for $c^{2}<a p$, a relation that always holds for satellite orbits. Thus (7) is satisfied if and only if

where

$$
b_{1} / \rho<g(\lambda)
$$

We shall next show that

$$
g(\lambda) \equiv \lambda^{2}-\lambda\left(\lambda^{2}-1\right)^{\frac{1}{2}}
$$

$$
1 / 2<g(\lambda) \leqq 1 \quad(\lambda \geqq 1) .
$$

To show that $g(\lambda) \leqq 1$, note that for $\lambda \geqq 1$, we have $1>1-\lambda^{-2} \geqq 0$, so that $\left(1-\lambda^{-2}\right)^{\frac{1}{2}} \geqq 1$ $-\lambda^{-2}, 1-\left(1-\lambda^{-2}\right)^{\frac{1}{2}} \leqq \lambda^{-2}$, and finally $\lambda^{2}-\lambda\left(\lambda^{2}-1\right)^{\frac{1}{2}} \leqq 1$. Thus $g(\lambda) \leqq 1$.

To show that $g(\lambda)>1 / 2$, note that for $\lambda \geqq 1$

so that

$$
0 \leqq 1-\lambda^{-2}<\left(1-\frac{1}{2} \lambda^{-2}\right)^{2},
$$

$$
\left(1-\lambda^{-2}\right)^{\frac{1}{2}}<1-\frac{1}{2} \lambda^{-2}
$$

or

Then

$$
1-\left(1-\lambda^{-2}\right)^{\frac{1}{2}}>\frac{1}{2} \lambda^{-2}
$$

$$
g(\lambda) \equiv \lambda^{2}-\lambda\left(\lambda^{2}-1\right)^{\frac{1}{2}}>1 / 2,
$$

as was to be shown.

From (9) and (11) it follows that when $\lambda \geqq 1$ the condition $b_{1} / \rho<1$ is necessary for the validity of (7) and that the condition $b_{1} / \rho<1 / 2$ is sufficient for its validity.

To show that $b_{1} / \rho<1 / 2$ for all bound ${ }^{4}$ orbits, note that $\rho \geqq \rho_{1}$, so that

$$
b_{1} / \rho \leqq b_{1} / \rho_{1} .
$$

From the relation $c^{2}=k p^{2}$ and the relation $\rho_{1}=a(1-e), e \leqq 1$, for a bound orbit, we then find from $(3.25),(4.12)$, and (5.6) that

$$
\frac{b_{1}}{\rho_{1}}=\frac{k(1+e)\left(1-\eta_{0}^{2}\right)\left[1-k \eta_{0}^{2}\left(1-e^{2}\right)\right]}{\left[1-k\left(1-e^{2}\right)\right]\left[1-k \eta_{0}^{2}\left(1-e^{2}\right)\right]+4 k \eta_{0}^{2}} .
$$

Here the numerator $\leqq k(1+e)$ and the denominator $\geqq(1-k)^{2}$, so that

$$
\frac{b_{1}}{\rho_{1}} \leqq \frac{k(1+e)}{(1-k)^{2}} \leqq \frac{2 k}{(1-k)^{2}}
$$

a function monotonic in $k$ for $0<k<1$ and $<1 / 2$ whenever $k<3-2 \sqrt{2}(=0.172)$.

For the earth $k<0.00108$, so that

$$
b_{1} / \rho_{1}<0.00216<1 / 2 .
$$

From (16) and (19) it follows that for the earth

$$
b_{1} / \rho<1 / 2 .
$$

\footnotetext{
4 Past participle of the verb "to bind," taken from the terminology of atomic theory.
} 
This is the condition sufficient for the validity of (7) and thus of the expansion (6), for all $\lambda \geqq 1$.

All the developments of (A) then hold for the $\rho$-integrals, in particular (5.30) through (5.33) for the integral $R_{1}$, (5.35) through (5.40) for $R_{2}$, and (5.60) through (5.65) for $R_{3}$, where the $D_{m}$ 's are again given by (5.50) and (5.53).

To show the rapid convergence of the various series that there occur, we note first that since

$$
P_{n}(\lambda)=\pi^{-1} \int_{0}^{\pi}\left[\lambda+\left(\lambda^{2}-1\right)^{1 / 2} \cos x\right]^{n} d x
$$

and since $\lambda \equiv b_{1} / b_{2} \geqq 1$, we have

$$
\left(b_{2} / p\right)^{n} P_{n}(\lambda)=\pi^{-1} \int_{0}^{\pi}\left[b_{1} p^{-1}+p^{-1}\left(b_{1}^{2}-b_{2}^{2}\right)^{1 / 2} \cos x\right]^{n} d x .
$$

Then

$$
\left|\left(\frac{b_{2}}{p}\right)^{n} P_{n}(\lambda)\right| \leqq\left[\frac{b_{1}}{p}+\frac{b_{1}}{p}\left(1-\lambda^{-2}\right)^{1 / 2}\right]^{n} \leqq\left(\frac{2 b_{1}}{p}\right)^{n}
$$

From (5.14) and (5.34) the series $S_{1}$ and $S_{2}$ that occur in the expressions for the $\rho$-integrals $R_{1}$ and $R_{2}$ are

$$
S_{j} \equiv \sum_{n=n_{j}}^{\infty}\left(\frac{b_{2}}{p}\right)^{n} P_{n}(\lambda) \int_{0}^{v}(1+e \cos x)^{n-n_{j}} d x, \quad(j=1,2)
$$

where $n_{1}=2$ and $n_{2}=0$. Thus

$$
\begin{aligned}
\left|S_{j}\right| & \leqq v \sum_{n=n_{j}}^{\infty}\left(\frac{2 b_{1}}{p}\right)^{n}(1+e)^{n-n_{j}} \\
& \leqq v \sum_{n=0}^{\infty}\left(\frac{2 b_{1}}{p}\right)^{n+n_{j}}(1+e)^{n} \leqq v\left(\frac{2 b_{1}}{p}\right)^{n_{j}} \sum_{n=0}^{\infty}\left[\frac{2 b_{1}}{p}(1+e)\right]^{n} \\
& \leqq \frac{v\left(\frac{2 b_{1}}{p}\right)^{n_{j}}}{1-\frac{2 b_{1}}{p}(1+e)} \quad(j=1,2) .
\end{aligned}
$$

But $p=a\left(1-e^{2}\right)=\rho_{1}(1+e)$, so that by (18)

$$
b_{1} p^{-1} \leqq k(1-k)^{-2}
$$

and

$$
2 b_{1}(1+e) p^{-1} \leqq 2 k(1+e)(1-k)^{-2} \leqq 4 k(1-k)^{-2},
$$

where $4 k(1-k)^{-2}<0.0043$ for the earth. It follows that the series for $R_{1}$ and $R_{2}$, and thus the series for the secular coefficients $A_{1}$ and $A_{2}$, converge absolutely and more rapidly than a geometric series of common ratio 0.0043 . $R_{3}$ is

By (5.49), (5.50), and (5.53) the series $S_{3}$ that occurs in the expression for the $\rho$-integral

$$
\begin{aligned}
& S_{3}=S_{e}+S_{o}, \\
& S_{e} \equiv \sum_{n=0}^{\infty} D_{2 n} \int_{0}^{v}(1+e \cos x)^{2 n+2} d x \\
& S_{o} \equiv \sum_{n=0}^{\infty} D_{2 n+1} \int_{0}^{v}(1+e \cos x)^{2 n+3} d x
\end{aligned}
$$




$$
\begin{aligned}
D_{2 n} & \equiv \sum_{j=0}^{n}(-1)^{n-j}(c / p)^{2 n-2 j}\left(b_{2} / p\right)^{2 j} P_{2 j}(\lambda) \\
D_{2 n+1} & \equiv \sum_{j=0}^{n}(-1)^{n-j}(c / p)^{2 n-2 j}\left(b_{2} / p\right)^{2 j+1} P_{2 j+1}(\lambda) .
\end{aligned}
$$

From (33) and (23) and the relation $c^{2}=k p^{2}$, it follows that

$$
\begin{aligned}
\left|D_{2 n}\right| \leqq k^{n} \sum_{j=0}^{n}\left(2 b_{1} / c\right)^{2 j} & \leqq k^{n} \sum_{j=0}^{\infty}\left(2 b_{1} / c\right)^{2 j} \\
& \leqq \frac{k^{n}}{1-\left(2 b_{1} / c\right)^{2}}
\end{aligned}
$$

and from (34) and (23) that

$$
\begin{aligned}
\left|D_{2 n+1}\right| \leqq k^{n}\left(2 b_{1} / p\right) \sum_{i=0}^{n}\left(2 b_{1} / c\right)^{2 j} & \leqq k^{n}\left(2 b_{1} / p\right) \sum_{j=0}^{\infty}\left(2 b_{1} / c\right)^{2 j} \\
& \leqq \frac{k^{n}\left(2 b_{1} / p\right)}{1-\left(2 b_{1} / c\right)^{2}}
\end{aligned}
$$

Thus, by (31) and (35)

and by (32) and (36) that

$$
\begin{aligned}
\left|S_{e}\right| & \leqq \frac{(1+e)^{2} v}{1-\left(2 b_{1} / c\right)^{2}} \sum_{n=0}^{\infty}\left[k(1+e)^{2}\right]^{n} \\
& \leqq \frac{(1+e)^{2} v}{\left[1-\left(2 b_{1} / c\right)^{2}\right]\left[1-k(1+e)^{2}\right]}
\end{aligned}
$$

$$
\begin{aligned}
\left|S_{o}\right| & \leqq \frac{(1+e)^{3} v\left(2 b_{1} / p\right)}{\left[1-\left(2 b_{1} / c\right)^{2}\right]} \sum_{n=0}^{\infty}\left[k(1+e)^{2}\right]^{n} \\
& \leqq \frac{(1+e)^{3} v\left(2 b_{1} / p\right)}{\left[1-\left(2 b_{1} / c\right)^{2}\right]\left[1-k(1+e)^{2}\right]} \cdot
\end{aligned}
$$

Then, by (30), (37), and (38), we have

$$
\left|S_{3}\right| \leqq \frac{(1+e)^{2} v\left[1+(1+e)\left(2 b_{1} / p\right)\right]}{\left[1-\left(2 b_{1} / c\right)^{2}\right]\left[1-k(1+e)^{2}\right]}
$$

But $p=\rho_{1}(1+e)$, so that by (18)

and

$$
2 b_{1} / p \leqq 2 k(1-k)^{-2}
$$

Thus

$$
2 b_{1} / c \equiv\left(2 b_{1} / p\right) k^{-1 / 2} \leqq 2 k^{1 / 2}(1-k)^{-2} \text {. }
$$

$$
\left|S_{3}\right| \leqq \frac{(1+e)^{2} v\left[1+2 k(1+e)(1-k)^{-2}\right]}{\left[1-4 k(1-k)^{-4}\right]\left[1-k(1+e)^{2}\right]}
$$

The series for $S_{3}$ thus converges absolutely and more rapidly than the power series in $k$ of the function on the right side of (42), where $k<0.0011$ for the earth. On replacing $v$ by $\pi$, we can then say the same thing about the secular coefficient $A_{3}$.

\section{Simplification of the $\rho$-Coefficients When $\lambda \geqq 1$}

From $\lambda=b_{1} / b_{2}$ it follows that

$$
b_{2}=b_{1} \lambda^{-1} \leqq b_{1} \quad(\lambda \geqq 1) .
$$

Since $b_{1}=O(k)$, it then follows that $b_{2}$ is also of order $k$ when $\lambda \geqq 1$. This fact enables us to simplify the coefficients $A_{1 n}$ and $A_{2 n}$, which are needed only to $O\left(k^{2}\right)$, and the coefficients $A_{3 n}$, which are needed only to $O(k)$. 
Thus (5.32) and (5.33) lead to

(5.39) and (5.40) lead to

$$
A_{11}=O\left(k^{3}\right) \quad A_{12}=O\left(k^{4}\right),
$$

$$
A_{23}=O\left(k^{3}\right) \quad A_{24}=O\left(k^{4}\right),
$$

and (5.37) and (5.38) lead to

$$
\begin{aligned}
& A_{21}=\left(1-e^{2}\right)^{\frac{1}{2}} p^{-1} e\left[b_{1} p^{-1}+\left(3-\lambda^{-2}\right) k^{2} \cos ^{4} I\right] \\
& A_{22}=\left(1-e^{2}\right)^{\frac{1}{2}} p^{-1} \frac{e^{2}}{8}\left(3-\lambda^{-2}\right) k^{2} \cos ^{4} I .
\end{aligned}
$$

Here we have used

in the terms involving. $b_{1}^{2} p^{-2}$.

$$
b_{1} p^{-1}=k \cos ^{2} I+O\left(k^{2}\right)
$$

Similarly, for the coefficients $A_{3 n}$, which are needed only to $O(k)$, we obtain from $(5.62)$ through (5.65)

$$
\begin{aligned}
& A_{31}=\left(1-e^{2}\right)^{\frac{1}{2}} p^{-3} e\left[2+\left(3+\frac{3}{4} e^{2}\right) k \cos ^{2} I-\left(4+3 e^{2}\right) k\right] \\
& A_{32}=\left(1-e^{2}\right)^{\frac{1}{2}} p^{-3} e^{2}\left[\frac{1}{4}+\frac{3}{4} k \cos ^{2} I-\left(\frac{e^{2}}{4}+\frac{3}{2}\right) k\right] \\
& A_{33}=\left(1-e^{2}\right)^{\frac{1}{2}} p^{-3} e^{3}\left[\frac{k}{12} \cos ^{2} I-\frac{k}{3}\right] \\
& A_{34}=-\left(1-e^{2}\right)^{\frac{1}{2}} p^{-3} e^{4} k / 32 .
\end{aligned}
$$

For $\lambda \geqq 1$ we need also to rewrite (5.31), (5.36), (5.50), and (5.53) for the secular coefficients $A_{1}, A_{2}, D_{2 n}$, and $D_{2 n+1}$, which all contain terms of the form $\left(b_{2} / p\right)^{m} P_{m}(\lambda)$. As $\sin I$ approaches zero, $b_{2}$ also approaches zero and $\lambda$ becomes infinite, so that such a term takes the indeterminate form zero times infinity. To remove this indeterminacy, note that

so that

$$
\frac{b_{2}}{p} \equiv \frac{b_{2}}{b_{1}} \frac{b_{1}}{p} \equiv \frac{b_{1}}{p} \lambda^{-1},
$$

$$
\begin{aligned}
\left(\frac{b_{2}}{p}\right)^{m} P_{m}(\lambda) & =\left(\frac{b_{1}}{p}\right)^{m} \lambda^{-m} P_{m}(\lambda) \\
& =\left(\frac{b_{1}}{p}\right)^{m} R_{m}\left(\lambda^{-1}\right) .
\end{aligned}
$$

Here $R_{m}(x)$ is a function that has already appeared in (A), viz,

$$
R_{m}(x) \equiv x^{m} P_{m}\left(x^{-1}\right)
$$

a polynomial of degree $[\mathrm{m} / 2]$ in $x^{2}$.

To determine $\left(b_{2} / p\right)^{m} P_{m}(\lambda)$ for $\sin I=0$, first write

$$
P_{m}(\lambda)=\sum_{j=0}^{[m / 2]} \frac{(-1)^{j}(2 m-2 j) ! \lambda^{m-2 j}}{2^{m} j !(m-j) !(m-2 j) !}
$$

so that

$$
\begin{aligned}
R_{m}\left(\lambda^{-1}\right) & \equiv \lambda^{-m} P_{m}(\lambda)=1 \quad(m=0,1) \\
& =\frac{(2 m) !}{2^{m}(m !)^{2}}+\sum_{j=1}^{[m / 2]} \frac{(-1)^{j}(2 m-2 j) ! \lambda^{-2 j}}{2^{m} j !(m-j) !(m-2 j) !} \quad(m=2,3,4, \ldots)
\end{aligned}
$$

Thus

$$
R_{m}(0)=\frac{(2 m) !}{2^{m}(m !)^{2}}, \quad(m=0,1,2,3, \ldots)
$$


so that by (55) and (59)

$$
\left(b_{2} / p\right)^{m} P_{m}(\lambda)=\frac{(2 m) !}{(m !)^{2}}\left(\frac{b_{10}}{2 p}\right)^{m}, \quad(\sin I=0)
$$

where $b_{10}$ is the value of $b_{1}$ for $\eta_{0}=\sin I=0$.

From (5.31), (5.36), (55), and (60) it then follows that

$$
\begin{aligned}
& A_{1}=\left(1-e^{2}\right)^{\frac{1}{2}} p \sum_{n=2}^{\infty}\left(b_{1} / p\right)^{n} R_{n}\left(\lambda^{-1}\right) R_{n-2}\left(\sqrt{1-e^{2}}\right) \quad(\lambda \geqq 1) \\
& =\left(1-e^{2}\right)^{\frac{1}{2}} p \sum_{n=2}^{\infty} \frac{(2 n) !}{(n !)^{2}}\left(\frac{b_{10}}{2 p}\right)^{n} R_{n-2}\left(\sqrt{1-e^{2}}\right) \quad(\sin I=0) \\
& A_{2}=\left(1-e^{2}\right)^{\frac{1}{2}} p^{-1} \sum_{n=0}^{\infty}\left(b_{1} / p\right)^{n} R_{n}\left(\lambda^{-1}\right) R_{n}\left(\sqrt{1-e^{2}}\right) \quad(\lambda \geqq 1) \\
& =\left(1-e^{2}\right)^{\frac{1}{2}} p^{-1} \sum_{n=0}^{\infty} \frac{(2 n) !}{(n !)^{2}}\left(\frac{b_{10}}{2 p}\right)^{n} R_{n}\left(\sqrt{1-e^{2}}\right) \quad(\sin I=0)
\end{aligned}
$$

Note that $A_{1}=O\left(k^{2}\right)$ and $A_{2}=O\left(k^{0}\right)$.

Then, from (5.61)

$$
A_{3}=\left(1-e^{2}\right)^{\frac{1}{2}} p^{-3} \sum_{m=0}^{\infty} D_{m} R_{m+2}\left(\sqrt{1-e^{2}}\right),
$$

where, by (5.50), (5.53), (55), (60), and the relation $c^{2}=k p^{2}$, we find

$$
\begin{aligned}
D_{2 n} & =\sum_{j=0}^{n}(-1)^{n-j}(c / p)^{2 n-2 j}\left(b_{2} / p\right)^{2 j} P_{2 j}(\lambda) \\
& =\sum_{j=0}^{n}(-1)^{n-j} k^{n-j}\left(b_{1} / p\right)^{2 j} R_{2 j}\left(\lambda^{-1}\right) \quad(\lambda \geqq 1) \\
& =\sum_{j=0}^{n}(-1)^{n-j} k^{n-j} \frac{(4 j) !}{[(2 j) !]^{2}}\left(\frac{b_{10}}{2 p}\right)^{2 j} \quad(\sin I=0) \\
D_{2 n+1} & =\sum_{j=0}^{n}(-1)^{n-j}(c / p)^{2 n-2 j}\left(b_{2} / p\right)^{2 j+1} P_{2 j+1}(\lambda) \\
& =\sum_{j=0}^{n}(-1)^{n-j} k^{n-j}\left(b_{1} / p\right)^{2 j+1} R_{2 j+1}\left(\lambda^{-1}\right) \quad(\lambda \geqq 1) \\
& =\sum_{j=0}^{n}(-1)^{n-j} k^{n-j} \frac{(4 j+2) !}{[(2 j+1) !]^{2}}\left(\frac{b_{10}}{2 p}\right)^{2 j+1} \quad(\sin I=0)
\end{aligned}
$$

\section{Simplification of Other Coefficients When $\lambda \geqq 1$}

To simplify the $\eta$-coefficients when $\lambda \geqq 1$, we must first note that from page (176) of (A) we have

so that

$$
b_{1} \approx k p \cos ^{2} I \quad b_{2} \approx k^{\frac{1}{2}} p \sin I,
$$

or

$$
\lambda \equiv b_{1} / b_{2} \approx k^{\frac{1}{2}} \cos ^{2} I \csc I
$$

Thus

$$
\eta_{0} \equiv \sin I \approx k^{\frac{1}{2}} \lambda^{-1} \cos ^{2} I .
$$

$$
\eta_{0}=O\left(k^{\frac{1}{2}}\right) \quad(\lambda \geqq 1) .
$$


Also, since $\eta_{2}^{-1}=O\left(k^{\frac{1}{2}}\right)$, by (3.42), it follows that

$$
q \equiv \eta_{0} / \eta_{2}=O(k) \quad(\lambda \geqq 1) .
$$

There also arises an indeterminacy in the $\eta$-coefficients, from the quantity $\left(\alpha_{2}^{2}-\alpha_{3}^{2}\right)^{-\frac{1}{2}} \eta_{0}$, which takes the form infinity times zero as $\eta_{0} \equiv \sin I$ approaches zero. To remove this indeterminacy, use (4.15a), viz,

to find

$$
\alpha_{3}=\alpha_{2}\left(1-\frac{c^{2} \eta_{0}^{2}}{a_{0} p_{0}}\right)^{1 / 2} \cos I
$$

$$
\left(\alpha_{2}^{2}-\alpha_{3}^{2}\right)^{-1 / 2} \eta_{0}=\alpha_{2}^{-1}\left(1+\frac{c^{2}}{a_{0} p_{0}} \cos ^{2} I\right)^{-1 / 2}
$$

Then, from (8.27) and (7.23)

$$
\begin{gathered}
\psi_{s}=2 \pi \nu_{2}\left[t+\beta_{1}+\beta_{2} \alpha_{2}^{-1}\left(a+b_{1}+A_{1}\right) A_{2}^{-1}\right] \\
2 \pi \nu_{2}=\alpha_{2}\left(1+\frac{c^{2}}{a_{0} p_{0}} \cos ^{2} I\right)^{1 / 2} A_{2} B_{2}^{-1}\left(a+b_{1}+A_{1}+c^{2} \eta_{0}^{2} A_{2} B_{1} B_{2}^{-1}\right)^{-1} .
\end{gathered}
$$

From (8.33)

$$
\psi_{0}=\left(-2 \alpha_{1}\right)^{-1 / 2} \alpha_{2}\left(1+\frac{c^{2}}{a_{0} p_{0}} \cos ^{2} I\right)^{1 / 2} A_{2} B_{2}^{-1} v_{0}
$$

From (8.37)

$M_{1}=\left(a+b_{1}\right)^{-1}\left[-\left(A_{1}+c^{2} \eta_{0}^{2} A_{2} B_{1} B_{2}^{-1}\right) v_{0}+\frac{c^{2}}{4}\left(-2 \alpha_{1}\right)^{\frac{1}{2}} \alpha_{2}^{-1}\left(1+\frac{c^{2}}{a_{0} p_{0}} \cos ^{2} I\right)^{-\frac{1}{2}} \eta_{0}^{2} \sin \left(2 \psi_{s}+2 \psi_{0}\right)\right]$,

of order $k^{2}$. Then, by (8.39)

$$
E_{1}=\left[1-e^{\prime} \cos \left(M_{s}+E_{0}\right)\right]^{-1} M_{1}
$$

since $M_{1}^{2}=O\left(k^{4}\right)$. Then $E_{1}$ and $v_{1}$ are both of order $k^{2}$. Also, by (8.40),

$$
\begin{aligned}
\psi_{1}=\left(-2 \alpha_{1}\right)^{-\frac{1}{2}} \alpha_{2}\left(1+\frac{c^{2}}{a_{0} p_{0}} \cos ^{2} I\right)^{\frac{1}{2}} B_{2}^{-1}\left[A_{2} v_{1}+A_{21} \sin \left(M_{s}+v_{0}\right)\right. & \\
& \left.+A_{22} \sin \left(2 M_{s}+2 v_{0}\right)\right]+\frac{q^{2}}{8} B_{2}^{-1} \sin \left(2 \psi_{s}+2 \psi_{0}\right) .
\end{aligned}
$$

By (8.45), $M_{2}$ and thus $E_{2}$ and $v_{2}$ are of order $k^{3}$ and, by (8.48), $\psi_{2}$ is also of order $k^{3}$. Thus all the second-order periodic terms of (A) become of the third order and thus negligible, when $\lambda \geqq 1$.

By (8.50) the right ascension $\varphi$ becomes

$$
\begin{aligned}
& \varphi=\beta_{3}+\alpha_{3} \alpha_{2}^{-1}\left(1+\frac{c^{2}}{a_{0} p_{0}} \cos ^{2} I\right)^{-\frac{1}{2}}\left[\left(1-\eta_{0}^{2}\right)^{-\frac{1}{2}}\left(1-\eta_{2}^{-2}\right)^{-\frac{1}{2}} \chi+B_{3} \psi\right] \\
&-c^{2} \alpha_{3}\left(-2 \alpha_{1}\right)^{-\frac{1}{2}}\left(A_{3} v+\sum_{n=1}^{4} A_{3 n} \sin n v\right) .
\end{aligned}
$$

Here we have dropped the periodic term $(3 / 32) \eta_{0}^{2} \eta_{2}^{-4} \sin 2 \psi$ of $(8.50)$, since it is of order $k^{3}$ for $\lambda \geqq 1$.

\section{Summary for $\lambda \geqq 1$}

For an almost equatorial orbit, corresponding to $\lambda \geqq 1$, the right ascension $\varphi$ is given by (83). The spheroidal coordinates $\rho$ and $\eta$ are given by

$$
\begin{aligned}
\rho=a(1-e \cos E) & =(1+e \cos v)^{-1} p \\
\eta & =\eta_{0} \sin \psi .
\end{aligned}
$$


Here the expressions

$$
E=M_{s}+E_{0}+E_{1} \quad v=M_{s}+v_{0}+v_{1} \quad \psi=\psi_{s}+\psi_{0}+\psi_{1}
$$

are sufficiently accurate to give the secular terms exactly and the periodic terms correctly through order $k^{2}$, provided that $M_{s}$ is calculated by (8.24), $\psi_{s}$ by (77), $E_{0}$ by (8.31), $E_{1}$ by (80) and $(81), v_{0}$ and $v_{1}$ by the anomaly relations (8.1), $\psi_{0}$ by (79), and $\psi_{1}$ by $(82)$.

\section{The Case of a Purely Equatorial Orbit, $I=0^{\circ}$ or $180^{\circ}$}

For $I=0^{\circ}$ or $180^{\circ}$ we have $\eta_{0}=0, \cos ^{2} I=1, \chi=\psi$ by $(6.51), \gamma_{m}=0$ by $(6.66)$,

by (6.65), and $\left|\alpha_{3}\right|=\alpha_{2}$, so that

$$
B_{3}=1-\left(1-\eta_{2}^{-2}\right)^{-\frac{1}{2}}
$$

Then, by (83),

$$
\alpha_{3} / \alpha_{2}=\operatorname{sgn} \alpha_{3} \text {. }
$$

$$
\varphi=\beta_{3}+\left(\operatorname{sgn} \alpha_{3}\right)\left(1+\frac{c^{2}}{a_{0} p_{0}}\right)^{-\frac{1}{2}} \psi-c^{2} \alpha_{3}\left(-2 \alpha_{1}\right)^{-\frac{1}{2}}\left(A_{3} v+\sum_{n=1}^{4} A_{3 n} \sin n v\right) .
$$

Also, by (8.24) with $\eta_{0}=0$,

$$
M_{s}=(-2 \alpha)^{\frac{1}{2}}\left(a+b_{1}+A_{1}\right)^{-1}\left(t+\beta_{1}\right)
$$

and by (77) and (78), with $\eta_{0}=0, \cos ^{2} I=1$, and $B_{2}=1$,

$$
\psi_{s}=\beta_{2}\left(1+\frac{c^{2}}{a_{0} p_{0}}\right)^{\frac{1}{2}}+\alpha_{2} A_{2}\left(1+\frac{c^{2}}{a_{0} p_{0}}\right)^{\frac{1}{2}}\left(a+b_{1}+A_{1}\right)^{-1}\left(t+\beta_{1}\right) .
$$

Thus, by (87) and (88),

$$
\psi_{s}=\beta_{2}\left(1+\frac{c^{2}}{a_{0} p_{0}}\right)^{\frac{1}{2}}+\alpha_{2}\left(1+\frac{c^{2}}{a_{0} p_{0}}\right)^{\frac{1}{2}}\left(-2 \alpha_{1}\right)^{-\frac{1}{2}} A_{2} M_{s} .
$$

Then, by (79) and (82), with $\eta_{0}=0$,

$$
\begin{aligned}
& \psi_{0}=\alpha_{2}\left(1+\frac{c^{2}}{a_{0} p_{0}}\right)^{\frac{1}{2}}\left(-2 \alpha_{1}\right)^{-\frac{1}{2}} A_{2} v_{0} \\
& \psi_{1}=\alpha_{2}\left(1+\frac{c^{2}}{a_{0} p_{0}}\right)^{\frac{1}{2}}\left(-2 \alpha_{1}\right)^{-\frac{1}{2}}\left[A_{2} v_{1}+\sum_{n=1}^{2} A_{2 n} \sin \left(n M_{s}+n v_{0}\right)\right] .
\end{aligned}
$$

Addition of (89) through (91) then gives

$$
\psi=\beta_{2}\left(1+\frac{c^{2}}{a_{0} p_{0}}\right)^{\frac{1}{2}}+\alpha_{2}\left(1+\frac{c^{2}}{a_{0} p_{0}}\right)^{\frac{1}{2}}\left(-2 \alpha_{1}\right)^{-\frac{1}{2}}\left[A_{2} v+\sum_{n=1}^{2} A_{2 n} \sin \left(n M_{s}+n v_{0}\right)\right] .
$$

Since $v=M_{s}+v_{0}+v_{1}$, where $v_{1}$ is of order $k^{2}$, and since $A_{21}$ and $A_{22}$ are of orders $k$ and $k^{2}$ respectively, it follows that

$$
\sum_{n=1}^{2} A_{2 n} \sin \left(n M_{s}+n v_{0}\right)=\sum_{n=1}^{2} A_{2 n} \sin n v
$$

to order $k^{2}$. Thus, to order $k^{2}$,

$$
\psi=\beta_{2}\left(1+\frac{c^{2}}{a_{0} p_{0}}\right)^{\frac{1}{2}}+\alpha_{2}\left(1+\frac{c^{2}}{a_{0} p_{0}}\right)^{\frac{1}{2}}\left(-2 \alpha_{1}\right)^{-\frac{1}{2}}\left(A_{2} v+\sum_{n=1}^{2} A_{2 n} \sin n v\right) .
$$

On inserting (94) into (86), we then find

$\phi=\beta_{3}+\beta_{2} \operatorname{sgn} \alpha_{3}+\alpha_{3}\left(-2 \alpha_{1}\right)^{-\frac{1}{2}}\left(A_{2} v+\sum_{n=1}^{2} A_{2 n} \sin n v\right)-c^{2} \alpha_{3}\left(-2 \alpha_{1}\right)^{-\frac{1}{2}}\left(A_{3} v+\sum_{n=1}^{4} A_{3 n} \sin n v\right)$. 
It is a simple exercise to check these results for a purely equatorial orbit. To do so, let $X$ and $Y$ be the usual Cartesian coordinates, define $\rho$ and $\phi$ by

$$
X+i Y=\left(\rho^{2}+c^{2}\right)^{\frac{1}{2}} \exp i \phi,
$$

write down the kinetic energy $\frac{1}{2}\left(\dot{X}^{2}+\dot{Y}^{2}\right)$ in terms of $\rho$ and $\phi$ and their time derivatives, write the potential as $-\mu \rho^{-1}$, construct the Hamiltonian, and then write down the Hamilton-Jacobi equation. Separate the latter, to obtain the solution

$$
W=\alpha_{3} \phi \pm \int_{\rho_{1}}^{\rho}\left(\rho^{2}+c^{2}\right)^{-1} F(\rho)^{\frac{1}{2}} d \rho,
$$

where

$$
F(\rho) \equiv c^{2} \alpha_{3}^{2}+\left(\rho^{2}+c^{2}\right)\left(-\alpha_{3}^{2}+2 \mu \rho+2 \alpha_{1} \rho^{2}\right) .
$$

The kinetic equations are then

$$
\begin{aligned}
\frac{\partial W}{\partial \alpha_{1}} & =t+\beta_{1}= \pm \int_{\rho_{1}}^{\rho} \rho^{2} F(\rho)^{-\frac{1}{2}} d \rho \\
\frac{\partial W}{\partial \alpha} & =\beta_{3}^{\prime}=\phi \mp \alpha_{3} \int_{\rho_{1}}^{\rho}\left(\rho^{2}+c^{2}\right)^{-1} \rho^{2} F(\rho)^{-\frac{1}{2}} d \rho \\
& =\phi \mp \alpha_{3} \int_{\rho_{1}}^{\rho} F(\rho)^{-\frac{1}{2}} d \rho \pm c^{2} \alpha_{3} \int_{\rho_{1}}^{\rho}\left(\rho^{2}+c^{2}\right)^{-1} F(\rho)^{-\frac{1}{2}} d \rho .
\end{aligned}
$$

On then following the procedure in $(\mathrm{A})$, we find that $\rho$ is given by just the results of section 5 of the present paper, with $\eta_{0}$ placed equal to zero, and that $\phi$ is given by

$$
\phi=\beta_{3}^{\prime}+\alpha_{3}\left(-2 \alpha_{1}\right)^{-\frac{1}{2}}\left(A_{2} v+\sum_{n=1}^{2} A_{2 n} \sin n v\right)-c^{2} \alpha_{3}\left(-2 \alpha_{1}\right)^{-\frac{1}{2}}\left(A_{3} v+\sum_{n=1}^{4} A_{3 n} \sin n v\right) .
$$

In order to reconcile (102) and (95), observe that for an equatorial orbit $\beta_{2}$ and $\beta_{3}$ are not defined separately, but enter in the combination $\beta_{3}+\beta_{2} \operatorname{sgn} \alpha_{3}$. Accordingly, the definition

$$
\beta_{3}^{\prime}=\beta_{3}+\beta_{2} \operatorname{sgn} \alpha_{3}
$$

will bring (102) and (95) into perfect agreement. To understand this definition better, we reason as follows.

In the equatorial plane $r^{2}=\rho^{2}+c^{2}$, so that $r$ is at minimum whenever $\rho$ is at minimum. That is, the satellite is at perigee whenever $v=2 \pi \tau, \tau=0,1,2, \ldots$ By (102) the right ascension $\phi_{p}$ thus changes value from one perigee to the next. For an equatorial orbit about a planet of zero oblateness, however, the coefficient of $v$ in (102), viz, $\alpha_{3}\left(-2 \alpha_{1}\right)^{-\frac{1}{2}}\left(A_{2}-c^{2} A_{3}\right)$, would reduce to $\operatorname{sgn} \alpha_{3}$, so that in such a limiting case

$$
\phi_{p}=\beta_{3}^{\prime}+2 \pi \tau \operatorname{sgn} \alpha_{3},(\tau=0,1,2, \ldots)
$$

so that the actual position of perigee would remain fixed and $\beta_{3}^{\prime}$ would be its right ascension.

But for the case of a nonequatorial orbit around a planet of zero oblateness $\beta_{3}$ is simply the right ascension $\Omega$ of the ascending node and $\beta_{2}$ is the argument $\omega$ of perigee. Thus for the limiting case of a purely equatorial orbit about a planet of zero oblateness (103) would take the expected form

$$
\text { R.A. of perigee }=\Omega \pm \omega \text {, }
$$

where the sign would be plus for a direct orbit and minus for a retrograde orbit. Thus the definition (103) is reasonable.

(Paper 66B1-68) 